\title{
Asymptotic violation of Bell inequalities and distillability
}

\author{
Lluís Masanes \\ School of Mathematics, University of Bristol, Bristol BS8 1TW, U.K.
}

(Dated: October 22, 2018)

\begin{abstract}
A multipartite quantum state violates a Bell inequality asymptotically if, after jointly processing by general local operations an arbitrarily large number of copies of it, the result violates the inequality. In the bipartite case we show that asymptotic violation of the CHSH-inequality is equivalent to distillability. Hence, bound entangled states do not violate it. In the multipartite case we consider the complete set of full-correlation Bell inequalities with two dichotomic observables per site, also called WWZB-inequalities. We show that asymptotic violation of any of these inequalities by a multipartite state implies that pure-state entanglement can be distilled from it, although the corresponding distillation protocol may require that some of the parties join into several groups. We also obtain the extreme points of the set of distributions generated by measuring $N$ quantum systems with two dichotomic observables per site. It is shown that when considering the violation of any Bell inequality after preprocessing, either deterministic LOCC or stochastic local operations (without communication) is enough.
\end{abstract}

In 1964 Bell ruled out the possibility that a local classical theory could give the same predictions than Quantum Mechanics [1]. Bell's Theorem states that the probabilities for the outcomes obtained when suitably measuring some quantum states cannot be generated from classical correlations. This turns out to be a general feature for entangled pure states, as it was proven in [2] that, a multipartite pure states is entangled (not product) if, and only if, it is not simulable by a local variable model (LVM). The situation is more complex for mixed states. There are mixed states that, though being entangled, whenever a single copy of the state is measured there is always a LVM giving the same predictions $[3,4]$. But some of these states violate Bell inequalities if, prior to the measurement, the state is preprocessed [5]. The most general preprocessing consists of stochastic local operations and classical communication (SLOCC), that is, LOCC protocols that fail with some probability. In this paper it is shown that when considering violation of Bell inequalities, both: preprocessing by stochastic local operations without communication (SLO), and, preprocessing by (deterministic) LOCC, are completely general [6]. It is important to remark that, in order to build a consistent picture, the preprocessing (in particular the classical communication) has to be made before the parties choose the experimental settings for the Bell test.

Another way of making manifest the nonlocality "hidden" in a mixed state is by jointly measuring more than one copy of it. In [7] it is shown that some states having a LVM for the single copy scenario, violate Bell inequalities when jointly measuring more than one copy. Then, merging these two ideas, a strong test for detecting the nonlocality "hidden" in a state is to measure the result of jointly processing by SLO an arbitrarily large number of copies of the state (as mentioned above, SLO is completely general). If the resulting probabilities violate a Bell inequality, we say that the original state violates this inequality asymptotically. Clearly, this kind of test is more general than above mentioned ones. That is, the set of states that violate a Bell inequality asymptotically includes the states that violate it straightaway. Later, we discuss how general the notion of asymptotic nonlocality is, and relate it to distillability.

A bipartite state is said to be distillable if, from an arbitrarily large number of copies of it, some pure-state entanglement can be extracted by LOCC [8]. For states of more than two parties different notions of distillability can be considered. For instance, there are $N$-partite states which are undistillable when the $N$ parties remain separated, but if some of the parties join together, purestate entanglement among the different groups of parties can be obtained [9]. Here, we also say that such states are distillable.

Distillability and violation of Bell inequalities are two manifestations of entanglement. On one hand, distillability is related to the usefulness in quantum information processing tasks, due to the fact that most of them require pure-state entanglement as a principal ingredient. On the other hand, Bell violation expresses the fact that a state cannot be simulated by classical correlations. This seems to be also a requirement if we want that a quantum information task gives an advantage over its classical counterparts. In particular, violation of Bell inequalities is a necessary and sufficient condition for the usefulness of a quantum state in communication complexity tasks [10]. In this paper, the link between these two concepts is analyzed. In particular, it is shown that asymptotic violation of the CHSH-inequality [11] is equivalent to distillability.

One of the most remarkable results in the field of Bell inequalities is the complete characterization of all $N$-partite inequalities with full-correlation functions of two dichotomic observables per site $[12,13]$, here called WWZB-inequalities. A relation between distillability and violation of WWZB-inequalities for $N$-qubit sys- 
tems was presented in [14-16]. They showed that the violation of a WWZB-inequality by a multiqubit state implies that pure-state entanglement can be distilled from it, although the corresponding distillation protocol may require that some of the parties join into several groups. In this paper we generalize their results to $N$-partite systems with arbitrary local Hilbert spaces (instead of qubits). We also generalize these results to the asymptotic scenario. In particular, given an arbitrary $N$-partite state, asymptotic violation of a WWZBinequality implies distillability in the same sense as mentioned above.

Let us specify the scenario and the notation. Consider $N$ separated parties, denoted by $n=1, \ldots N$, each having a physical system which can be measured with one among $M$ observables with $K$ outcomes each. The $n^{\text {th }}$ party observables and outcomes are respectively denoted by $x_{n} \in\{1, \ldots M\}$ and $a_{n} \in\{1, \ldots K\}$. All the experimental information is in the joint probability distribution for the outcomes conditioned on the chosen observables $P\left(a_{1} \ldots a_{N} \mid x_{1} \ldots x_{N}\right)$. Distributions that correspond to LVM are the ones that can be written as

$$
P\left(a_{1} \ldots a_{N} \mid x_{1} \ldots x_{N}\right)=\sum_{\lambda} p(\lambda) \prod_{n=1}^{N} P_{n}\left(a_{n} \mid x_{n} \lambda\right)
$$

see [17]. Fixed $N, M, K$ to some finite values, the set of distributions $P$ that can be written as (1) is a convex polytope, which can be characterized by a finite set of linear inequalities [17]. Each of these inequalities, denoted $\beta$, is characterized by its coefficients

$$
\begin{aligned}
\beta[P]=\sum_{a_{1}, x_{1}} & \cdots \sum_{a_{N}, x_{N}} \beta\left(a_{1} \ldots a_{N} \mid x_{1} \ldots x_{N}\right) \\
& \times P\left(a_{1} \ldots a_{N} \mid x_{1} \ldots x_{N}\right) \geq 0 .
\end{aligned}
$$

By definition, all distributions of the form (1) satisfy (2). If one inequality $\beta$ is violated by some probability distributions, we say that $\beta$ is a Bell inequality.

Let us characterize the set of distributions that can be generated within quantum theory. Suppose the $n^{\text {th }}$ party has a system with Hilbert space $\mathcal{H}_{n}$, which is measured with the $M$ generalized measurements $\left\{A_{n}(a \mid x)\right.$ : $a=1, \ldots K\}$ for $x=1, \ldots M$. These POVMs satisfy $A_{n}(a \mid x) \geq 0$ for $a=1, \ldots K$ and $\sum_{a=1}^{K} A_{n}(a \mid x)=\mathbb{I}_{n}$, for $x=1, \ldots M$ and $n=1, \ldots N$, where $\mathbb{I}_{n}$ is the identity matrix acting on $\mathcal{H}_{n}$. The quantum distributions are the ones that can be written as

$$
P\left(a_{1} \ldots a_{N} \mid x_{1} \ldots x_{N}\right)=\operatorname{tr}\left[\rho \bigotimes_{n=1}^{N} A_{n}\left(a_{n} \mid x_{n}\right)\right],
$$

where $\rho$ is a positive semidefinite matrix acting on $\mathcal{H}=$ $\bigotimes_{n=1}^{N} \mathcal{H}_{n}$ with $\operatorname{tr} \rho=1$. It is shown in [12] that the sets of quantum distributions (3) are convex, but little is known about them. Here we obtain a characterization of all the extreme points for the case $K=M=2$ and arbitrary $N$.

Lemma. Let $A_{1}, A_{2}, B_{1}, B_{2}$ be four projectors acting on a Hilbert space $\mathcal{H}$ such that $A_{1}+A_{2}=\mathbb{I}$ and $B_{1}+B_{2}=\mathbb{I}$. There exists an orthonormal basis in $\mathcal{H}$ where the four projectors $A_{1}, A_{2}, B_{1}, B_{2}$ are simultaneously block-diagonal, in blocks of size $1 \times 1$ or $2 \times 2$.

proof. The three positive operators $B_{1},\left(B_{1} A_{1} B_{1}\right)$, $\left(B_{1} A_{2} B_{1}\right)$ can be simultaneously diagonalized, because their ranges are contained in the subspace where $B_{1}$ acts like the identity and $B_{1} A_{1} B_{1}+B_{1} A_{2} B_{1}=B_{1}$. Let $|v\rangle$ be one of their simultaneous eigenvectors which satisfies $B_{2}|v\rangle=\mathbf{0}$. Because $A_{1}+A_{2}=\mathbb{I}$, it cannot be the case that $A_{1}|v\rangle=A_{2}|v\rangle=\mathbf{0}$. If $A_{1}|v\rangle=\mathbf{0}$ then $A_{2}|v\rangle=B_{1}|v\rangle=|v\rangle$ and the span of $|v\rangle$ (denoted $E_{v}$ ) corresponds to a $1 \times 1$ diagonal block in which $A_{1}, A_{2}, B_{1}, B_{2}$ have eigenvalues $0,1,1,0$, respectively. The case $A_{2}|v\rangle=\mathbf{0}$ is similar. Consider the case where $A_{1}|v\rangle \neq \mathbf{0}$ and $A_{2}|v\rangle \neq \mathbf{0}$. Define the orthogonal vectors $\left|a_{1}\right\rangle=A_{1}|v\rangle,\left|a_{2}\right\rangle=A_{2}|v\rangle$, and the two-dimensional subspace $E_{v}=\left\{\alpha_{1}\left|a_{1}\right\rangle+\alpha_{2}\left|a_{2}\right\rangle: \forall \alpha_{1}, \alpha_{2} \in \mathbb{C}\right\}$. The fact $|v\rangle=\left|a_{1}\right\rangle+\left|a_{2}\right\rangle$ implies $|v\rangle \in E_{v}$. Because $B_{1}\left|a_{1}\right\rangle \propto|v\rangle$ and $B_{1}\left|a_{2}\right\rangle \propto|v\rangle$, there exists a vector $|w\rangle \in E_{v}$ such that $B_{1}|w\rangle=\mathbf{0}$ and $B_{2}|w\rangle=|w\rangle$. Summarizing, the vectors $\left|a_{1}\right\rangle,\left|a_{2}\right\rangle \in E_{v}$ are simultaneous eigenvectors of $A_{1}, A_{2}$, and the vectors $|w\rangle,|v\rangle \in E_{v}$ are simultaneous eigenvectors of $B_{1}, B_{2}$. Therefore, the subspace $E_{v}$ corresponds to a $2 \times 2$ simultaneous diagonal block for $A_{1}, A_{2}, B_{1}, B_{2}$. The same can be done with the rest of simultaneous eigenvectors $|v\rangle$ as defined above. And analogously, for the simultaneous eigenvectors of $B_{2},\left(B_{2} A_{1} B_{2}\right),\left(B_{2} A_{2} B_{2}\right)$ which are orthogonal to the vectors $|w\rangle$ that have appeared in the previous steps. At the end, the direct sum of the subspaces $E_{1}, E_{2} \ldots$ is $\mathcal{H}$, each subspace $E_{i}$ of dimension two contains two eigenvectors of each operator $A_{1}, A_{2}, B_{1}, B_{2}$.

Result 1. In the case $K=M=2$, all quantum extreme points (3) are achievable by measuring $N$-qubit pure states with projective observables.

Proof. It is easy to see that all two-outcome POVMs are mixtures of two-outcome projective measurements. Hence, the distribution (3) can be written as a mixture of distributions where the operators $A_{n}(a \mid x)$ are projectors. According to the lemma, the four operators $A_{n}(a \mid x)$ for $a, x=1,2$ can be simultaneously blockdiagonalized, in blocks of size $2 \times 2$, at most. Denote by $\left\{E_{1}^{n}, E_{2}^{n} \ldots\right\}$ the projectors onto the subspaces corresponding to these diagonal blocks, for the $n^{\text {th }}$ party. If the $n^{\text {th }}$ party performs the measurement $\left\{E_{i}^{n}\right\}_{i}$ before measuring $\{A(1 \mid x), A(2 \mid x)\}$, the result does not change. But, after performing $\left\{E_{i}^{n}\right\}_{i}$ the local system is contained in a two-dimensional subspace. Applying this to all parties, the distribution (3) becomes a mixture of 
distributions generated by measuring $N$-qubit systems with projective observables. To finish, recall that $\rho$ can be expressed as a mixture of pure states.

Result 2. If an $N$-partite state $\rho$ violates the Bell inequality $\beta$ (for $M=K=2$ ), then $\rho$ can be transformed by SLO into an $N$-qubit state $\tilde{\rho}$ that violates $\beta$ by an equal or larger amount.

Proof. If $\rho$ violates $\beta$ then it does so with projective observables, because these are more extremal. Following the argument of the previous proof, the correlations obtained from $\rho$, say (3), do not change if the $n^{\text {th }}$ party performs the measurement $\left\{E_{i}^{n}\right\}_{i}$ before measuring $A_{n}(a \mid x)$, for all $n$. The final distribution (3) becomes a mixture of distributions generated by the family of two-qubit states $\left(E_{i_{1}}^{1} \otimes \cdots \otimes E_{i_{N}}^{N}\right) \rho\left(E_{i_{1}}^{1} \otimes \cdots \otimes E_{i_{N}}^{N}\right)$. By convexity, at least one of these states violates $\beta$ by at least the same amount.

As mentioned above, the set of states that violate a Bell inequality after a general preprocessing (SLOCC) is strictly larger than the set of states that violate Bell straightaway [5]. It could also be the case that, the set of states that violate Bell after SLOCC is larger than the set of states that do so after SLO, or (deterministic) LOCC; due to the fact that these two classes of operations are more restricted. Remarkably, the three sets are equally powerful. Recall that some authors also consider Bell inequalities which are not facets of the LVM-polytope. This result does not hold for them.

Result 3. If a state violates a Bell-inequality $\beta$ after SLOCC, then it also violates $\beta$ after SLO or LOCC.

Proof. To see that SLO is enough, recall that any SLOCC protocol can be pictured as a tree where different branches correspond to different strings of exchanged messages, and the result of at least one branch must violate $\beta$. The local operations corresponding to this branch constitute a SLO protocol which also achieves this goal. To show that LOCC is enough, suppose that $\Omega$ is a SLOCC protocol that can be applied to the state $\rho$ with probability $\pi=\operatorname{tr}[\Omega(\rho)]$, and the state $\Omega(\rho)$ violates the Bell inequality $\beta$ when measured with the observables $\left\{A_{n}\left(a_{n} \mid x_{n}\right)\right\}$. Suppose that $P_{0}$ is a LVM distribution (1) that saturates the inequality, $\beta\left[P_{0}\right]=0$. Given $P_{0}$ one can always get a separable state $\rho_{0}$ and a set of observables $\left\{B_{n}\left(a_{n} \mid x_{n}\right)\right\}$ such that

$$
P_{0}\left(a_{1} \ldots a_{N} \mid x_{1} \ldots x_{N}\right)=\operatorname{tr}\left[\rho_{0} \bigotimes_{n=1}^{N} B_{n}\left(a_{n} \mid x_{n}\right)\right] .
$$

Define a LOCC protocol $\tilde{\Omega}$ in the following way. The parties perform the protocol $\Omega$ to $\rho$, if it succeeds each party prepares an ancillary system in the state $|0\rangle$, if it fails each party prepares an ancillary system in the state $|1\rangle$ and all together prepare the separable state $\rho_{0}$ (by LOCC), and finally, all parties forget the state of the ancillary systems. The state $\rho$ after this protocol is

$$
\tilde{\Omega}(\rho)=\pi \Omega(\rho) \otimes|0\rangle\left\langle\left. 0\right|^{\otimes N}+(1-\pi) \rho_{0} \otimes \mid 1\right\rangle\left\langle\left. 1\right|^{\otimes N} .\right.
$$

Clearly, if this state is measured with the observables $\left\{A_{n}\left(a_{n} \mid x_{n}\right) \otimes|0\rangle\left\langle 0\left|+B_{n}\left(a_{n} \mid x_{n}\right) \otimes\right| 1\right\rangle\langle 1|\right\}$ one obtains a strict violation of $\beta$, as we wanted to prove.

Let us move to the asymptotic scenario. In the rest of the paper we only consider the case where each party has two dichotomic observables $(M=K=2)$. From Result 2, straight conclusions can be obtained in the bipartite case.

Result 4. A bipartite state $\rho$ is distillable if, and only if, there exist a positive integer $m$ and a SLO map $\Omega$ such that $\Omega\left[\rho^{\otimes m}\right]$ violates CHSH.

Proof. By definition, if $\rho$ is distillable there exist an integer $m$ and a SLO map $\Omega$ such that the state $\Omega\left[\rho^{\otimes m}\right]$ is close enough to a singlet for violating $\mathrm{CHSH}$. Conversely, if there exists an integer $m$ and a SLO map $\Omega$ such that $\Omega\left[\rho^{\otimes m}\right]$ violates CHSH, by Result 2 , the state $\Omega\left[\rho^{\otimes m}\right]$ can be transformed by SLO into a two-qubit state $\tilde{\rho}$ which violates CHSH. Clearly, $\tilde{\rho}$ is entangled and thus distillable [18], therefore $\rho$ is distillable too.

Let us generalize this result to the multipartite case. When the observables are dichotomic $(K=2)$, one can reduce the amount of experimental data by considering full-correlation functions

$$
\begin{aligned}
& C\left(x_{1} \ldots x_{N}\right)= \\
& \sum_{a_{1}=1}^{2} \ldots \sum_{a_{N}=1}^{2}(-1)^{\sum_{n=1}^{N} a_{n}} P\left(a_{1} \ldots a_{N} \mid x_{1} \ldots x_{N}\right) .
\end{aligned}
$$

That is, for each experimental setting $\left(x_{1} \ldots x_{N}\right)$ all the information is summarized in the single number $C\left(x_{1} \ldots x_{N}\right)$, instead of the $2^{N}$ numbers $P\left(a_{1} \ldots a_{N} \mid x_{1} \ldots x_{N}\right)$. In the case where each party has two dichotomic observables $(M=K=2)$, the necessary and sufficient conditions for a given sample of correlators $\left\{C\left(x_{1} \ldots x_{N}\right)\right\}$ to be obtainable by a LVM are the WWZB-inequalities $[12,13]$. A link between violation of WWZB-inequalities and distillability for $N$-qubit systems was obtained in [14-16]. They proved that, if an $N$-qubit state $\rho$ violates a WWZB-inequality $\beta$ by an amount $\beta[\rho]$ such that

$$
1<2^{\frac{N-G-1}{2}}<\beta[\rho] \leq 2^{\frac{N-G}{2}},
$$

then pure-state entanglement can be extracted from $\rho$ when the parties join into groups of at most $G$ people. That is, the larger the violation is, the smaller the size of the groups $G$ has to be in order to distill.

Result 2 straightforwardly allows for generalizing this results to any $N$-partite state $\rho$, not necessarily composed of qubits. Also, following the same reasoning as 
in Result 4, one can generalize it to hold in the asymptotic scenario.

Result 5. Consider an $N$-partite state $\rho$, an integer $m$ and a SLO map $\Omega$ such that the WWZB-inequality $\beta$ is asymptotically violated by the amount $\beta\left[\Omega\left(\rho^{\otimes m}\right)\right]$ in the range

$$
1<2^{\frac{N-G-1}{2}}<\beta\left[\Omega\left(\rho^{\otimes m}\right)\right] \leq 2^{\frac{N-G}{2}} .
$$

Then, pure-state entanglement can be extracted from $\rho$ when the parties join into groups of at most $G$ people.

Discussion. When considering violations of Bell inequalities, the asymptotic scenario is more general than that with single-copy preprocessing [5], and joint measurements on few copies of the state [7]. However, there is another scenario which in principle is not included in the asymptotic one, namely, when sequences of more than one measurement in each site are applied to the same system [19]. Interestingly, the only known strategy to apply sequences of measurements [19] in order to violate Bell inequalities is equivalent to SLO-preprocessing of the state [20]. Then, the asymptotic scenario may be completely general when considering Bell violation with a single species of state.

We have seen that in the bipartite case, asymptotic nonlocality is equivalent to distillability, if we impose that the Bell experiment is made with two dichotomic observables per site $(K=M=2)$. As byproduct we obtain a new result: bound entangled states [21] do not violate $\mathrm{CHSH}$, even asymptotically. It would be very interesting to know whether the above equivalence holds without any restriction on $K$ and $M$. Unfortunately, the techniques used in the lemma cannot be directly extended to larger values of $K$ or $M$. In the multipartite case, we have generalized the results obtained in [14-16] to arbitrary states (not only multi-qubit states), and also for the asymptotic scenario.

There is another scenario which is more general than the one of asymptotic nonlocality. Namely, when a bipartite state cannot be simulated by classical correlations in all possible situations [22]. These include situations when the bipartite state $\rho$ is jointly processed with a different state $\omega$. In the case where $\omega$ is equal to $\rho^{\otimes n}$ we recover the asymptotic scenario. It is proven in [22] that a bipartite state is simulable by classical correlations in all possible situations if, and only if, it is separable. Merging this equivalence with the one of Result 4 an appealing picture for bipartite states emerges:

$$
\begin{aligned}
& \text { entangled } \Longleftrightarrow \text { nonsimulable in general } \\
& \text { distillable } \Longleftrightarrow \text { nonsimulable in the asymp. scen. }
\end{aligned}
$$

Unfortunately, the second equivalence is only proved for the case $K=M=2$. But as argued above, it could hold in general.
We have also studied the set of probability distributions that arise when measuring $N$ quantum systems with two dichotomic observables per site. We have shown that the extreme points of this convex set are obtainable by measuring $N$-qubit pure states with projective observables. This answers the question posed in Problem 26A of [23], for the case $M=K=2$. It would be very interesting to generalize this result by showing that, the minimal dimension of the local Hilbert space sufficient for generating all extreme quantum correlations in the setting $(N, M, K)$ is $K$. Unfortunately, the technique used in the lemma is not directly applicable for $M, K>2$. These bounds on the dimensionality of the local Hilbert space allow for using known algorithms [24] in order to decide whether some correlations are predictable by quantum theory or not. This could be used to falsate quantum mechanics without assuming any model for the experiment, as Bell inequalities do so for classical physics.

Acknowledgements. The author is thankful to Nick S. Jones for useful comments. This work has been supported by the U.K. EPSRC's "IRC QIP".

[1] J. S. Bell; Physics 1, 195 (1964).

[2] S. Popescu, D. Rohrlich; Phys. Lett. A 166, 293 (1992).

[3] R. F. Werner; Phys. Rev. A 40, 4277 (1989).

[4] J. Barrett; Phis. Rev. A 65, 042302 (2002).

[5] N. Gisin; Phys. Lett. A 210, 151 (1996).

[6] A deterministic operation is one that succeeds with probability one (trace-preserving).

[7] A. Peres; Phys. Rev. A 54, 2685 (1996).

[8] C. H. Bennett, D. P. DiVincenzo, J. A. Smolin, W. K. Wootters; Phys. Rev. A 54, 3824 (1996).

[9] W. Dür, J. I. Cirac; Phys. Rev. A 61, 062313 (2000).

[10] C. Brukner, M. Zukowski, J. W. Pan, A. Zeilinger; quant-ph/0210114.

[11] J. F. Clauser, M. A. Horne, A. Shimony, R. A. Holt; Phys. Rev. Lett. 23, 880 (1969).

[12] R. F. Werner, M. M. Wolf; Phys. Rev. A. 64, 032112.

[13] M. Zukowski, C. Brukner; Phys. Rev. Lett. 88, 210401.

[14] A. Acín; Phys. Rev. Lett. 88, 027901 (2002).

[15] A. Acín, V. Scarani, M. M. Wolf; J. Phys. A: Math. Gen. 36, L21 (2003).

[16] A. Acín, V. Scarani, M. M. Wolf; Phys. Rev. A 66, 042323 (2002).

[17] R. Werner, M. M. Wolf; quant-ph/0107093.

[18] M. Horodecki, P. Horodecki, R. Horodecki; Phys. Rev. Lett. 78, 574 (1997).

[19] S. Popescu; Phys. Rev. Lett. 74, 2619, (1995).

[20] A. Peres; quant-ph/9512003.

[21] M. Horodecki, P. Horodecki, R. Horodecki; Phys. Rev. Lett. 80, 5239 (1998).

[22] Ll. Masanes, A. Doherty, Y. Liang; In preparation.

[23] O. Krueger, R. F. Werner; quant-ph/0504166.

[24] M. Grötschel, L. Lovasz, G. Schrijver; Geometric Algorithms and Combinatorical Optimization (SpringerVerlag, Berlin 1988). 